\title{
Charged Pion Photoproduction
}

\author{
Wei Chen and Haiyan Gao \\ Department of Physics and the Triangle Universities Nuclear Laboratory, Duke University, \\ Durham, NC 27708, U.S.A
}

\begin{abstract}
We extracted the differential cross section for the $\gamma n \rightarrow \pi^{-} p$ process from a deuterium target using the CLAS detector at Jefferson Lab in Hall B for photon energies between 1.0 and 3.5 $\mathrm{GeV}$ and pion center-of-mass (c.m.) angles $\left(\theta_{c . m .}\right.$.) between $50^{\circ}$ and $115^{\circ}$. We confirm a previous indication of a broad enhancement around a c.m. energy $(\sqrt{s})$ of $2.1 \mathrm{GeV}$ at $\theta_{c . m .}=90^{\circ}$ in the scaled differential cross section, $s^{7} \frac{d \sigma}{d t}$ and a rapid fall-off in a center-of-mass energy region of about 400 $\mathrm{MeV}$ following the enhancement. Our data show an angular dependence of this enhancement as the

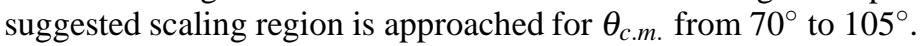

Keywords: pion photoproduction, constituent couting rule

PACS: 13.60.Le, 24.85.+p, 25.10.+s, 25.20.-x

\section{INTRODUCTION}

The $\gamma n \rightarrow \pi^{-} p, \gamma p \rightarrow \pi^{+} n$ and $\gamma p \rightarrow \pi^{0} p$ reactions are fundamental processes that are ideal candidates for the study of the strong interaction. At intermediate energies these processes have been used to study nucleon resonances and the transition from nucleon-meson to quark-gluon degrees of freedom. Recently at Jefferson Lab, the differential cross sections on the charged pion photoproduction on hydrogen (proton) and deuterium (deuteron) targets $[1,2]$ have been measured at intermediate energies. These measurements have shown that the differential cross section for pion photoproduction at fixed c.m. angles of $70^{\circ}$ and $90^{\circ}$ seem to scale as $d \sigma / d t \propto s^{-(n-2)}$ as predicted by the constituent counting rule (CCR) $[3,4]$. Here $s$ is the invariant Mandelstam variable for the total energy squared and $n$ is the total number of point particles and gauge fields involved. The CCR was proposed as a signature for the transition from the nucleonmeson to the quark-gluon picture. While such predicted scaling behavior has been seen in a number of exclusive reactions at specific kinematic regime $[5,6,7,8,9,10,11]$, questions remain such as why scaling seems to set in at a surprisingly low transverse momentum value above about $1.1 \mathrm{GeV} / \mathrm{c}[9,11]$. In addition, the recent charged pion photoproduction experiment $[1,2]$ also observed an apparent enhancement in the scaled differential cross section at $\theta_{\text {c.m. }}=90^{\circ}$ and at $\sqrt{s} \approx 1.8-2.5 \mathrm{GeV}$. Furthermore, just before the onset of scaling behavior, the scaled cross section drops by a factor of 4 in a very narrow c.m. energy range of few hundreds of $\mathrm{MeV}$ around $\sqrt{s}=2.5 \mathrm{GeV}$.

The lack of data in this energy region did not allow for a detailed investigation of either the true nature of the apparent scaling behavior or the observed enhancement, and the drop in the differential cross section.

In this paper, we present a measurement aimed at a mapping of the transition from one region to another and providing a detailed investigation of the observed enhancement and the drop in the differential cross section for the $\gamma n \rightarrow \pi^{-} p$ process [12]. This 
measurement was carried out over the range $\sqrt{s}$ from 1.8 to $2.8 \mathrm{GeV}$ with a very fine photon energy binning and a high statistical precision, using the Jefferson Lab CEBAF Large Acceptance Spectrometer (CLAS) [13] in Hall B.

\section{EXPERIMENT OVERVIEW \& DATA ANALYSIS}

The CLAS instrumentation was designed to provide large coverage of charged particles $\left(8^{\circ} \leq \theta \leq 140^{\circ}\right)$. It is divided into six sectors by six superconducting coils which generate a toroidal magnetic field. Each sector acts as an independent detection system that includes drift chambers (DC), Cerenkov counters (CC), scintillation counters (SC) and electromagnetic calorimeters (EC).

A 24-cm long liquid-deuterium target was employed with the target cell positioned $25 \mathrm{~cm}$ upstream from the CLAS nominal center. A tagged-photon beam generated by a $3.8-\mathrm{GeV}$ electron beam incident on a gold radiator with a radiation length of $10^{-4}$, corresponded to a maximum $\sqrt{s}$ of $2.8 \mathrm{GeV}$ for the process of interest. The event trigger required at least two charged particles in different sectors. About $10^{10}$ triggers were collected during a running period of about two months.

The events of interest were selected by detecting proton and $\pi^{-}$in coincidence. The momentum of the spectator proton in the deuteron is mostly below $200 \mathrm{MeV} / \mathrm{c}$ and is therefore not detected by CLAS. We identified this spectator using the missing mass technique. The quasifree process was selected by further applying a missing momentum cut of less than $200 \mathrm{MeV} / \mathrm{c}$.

To extract the cross section, a phase space based simulation is used to correct for events lost due to geometrical constraints and detector inefficiencies including the DC wire efficiency and SC efficiency. The final state interaction (FSI) effects have been taken into account before one extracts cross sections on the neutron since a deuteron target is used. The FSI correction is calculated according to the Glauber formulation [14] and this correction is about $15 \%-30 \%$ depending on energy and angle.

There are three major sources of systematic uncertainties: the luminosity, the FSI correction, and the background. The uncertainty of the luminosity was determined to be within $5 \%$. For the FSI, we carried out another calculation using the approach of Ref. [15] in addiction to the Glauber calculation.

Both methods agree within $10 \%$. A $10 \%$ systematic uncertainty to the differential cross section is assigned for the FSI correction. The background in the missing mass peak region is about $2 \%-7 \%$ depending on the photon energy. According to Monte Carlo simulations, the background could come from the poorly reconstructed real events due to the DC resolution. Therefore, the fitted background was assigned as the systematic uncertainty. The total systematic uncertianty is the quadratic sum of all the systematic uncertainties, and is between $11 \%$ to $13 \%$ on the extracted differential cross sections. 


\section{DIFFERENTIAL CROSS SECTIONS}

The differential cross sections agree with the world data very well. As shown in Fig. 1 (left panel), our data (red) are consistent with the E94-104 results [1] within experimental uncertainties. With fine photon energy bins and high statistical precision, our data confirm a broad enhancement around $\sqrt{s}$ of $2.1 \mathrm{GeV}$ in the scaled differential cross section. Our data also confirm a marked fall-off of the differential cross section in a narrow energy window of about $400 \mathrm{MeV}$ above this enhancement and the onset of the CCR scaling for $\sqrt{s}$ around $2.8 \mathrm{GeV}$ as suggested by an earlier Jefferson Lab experiment [1] (shown as green solid squares). Similar behavior has been seen in the recent CLAS g1c
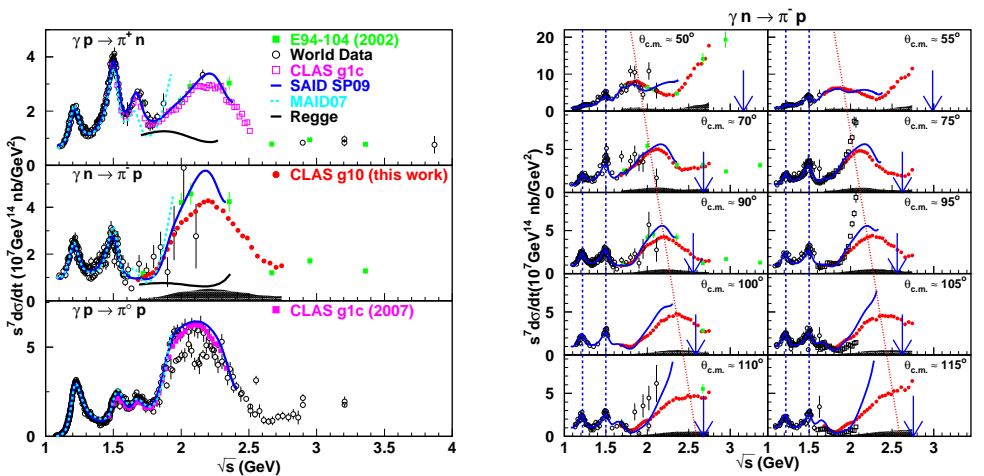

FIGURE 1. (color online). Scaled differential cross section $s^{7} \frac{d \sigma}{d t}$ as a function of $\sqrt{s}$. On the left are the scaled differential cross sections at $\theta_{c . m .}=90^{\circ}$ for three different channels. On the right are the scaled differential cross sections for $\theta_{c . m .}=50^{\circ}$ to $115^{\circ}$ for $\pi^{-}$channel.

$\pi^{+}$photoproduction data [16] (magenta). While this fall-off may be taken as a signature for the transition from nucleon-meson degrees of freedom to quark-gluon degrees of freedom, theoretical studies in this region are needed to confirm this speculation. Also shown are the results of the SAID SP09 partial wave analysis [16] (blue), the MAID07 model [17] (cyan), and the prediction from a Regge approach [18] (black).

In the Regge calculation, no baryon resonances in this energy region were included. And the results didn't predict the enhancement seen in our data. Thus the deviation is speculated to be due to baryon resonances [18].

Fig. 1 (right panel) shows the scaled differential cross section $s^{7} \frac{d \sigma}{d t}$ as a function of $\sqrt{s}$ for $\theta_{c . m .}=50^{\circ}$ to $115^{\circ}$ with an angular bin size of $5^{\circ}$ for the $\gamma n \rightarrow \pi^{-} p$ process. The blue arrows indicate the location of $\sqrt{s}$ corresponding to a pion transverse momentum $\left(p_{T}\right)$ of $1.1 \mathrm{GeV} / \mathrm{c}$. This $p_{T}$ value was suggested to govern the scaling onset by Refs. [9, 11]. An angular-dependent feature in the scaled differential cross section is clearly seen in our data. The aforementioned broad enhancement around a $\sqrt{s}$ value of $2.1 \mathrm{GeV}$ at $\theta_{c . m .}=90^{\circ}$ seems to shift as a function of $\theta_{\text {c.m. }}$ from $\sqrt{s}$ of $1.80 \mathrm{GeV}$ at $50^{\circ}$ to 2.45 $\mathrm{GeV}$ at $105^{\circ}$ as shown by the red dotted lines. Our studies show that such behavior is not an artifact of the $s^{7}$ scaling factor. It is not clear whether this enhancement dies off for $\theta_{c . m .}>105^{\circ}$ or whether it shifts to further higher energies. The blue dashed lines indicate the locations of the nucleon resonances around $1.2 \mathrm{GeV}$ and $1.5 \mathrm{GeV}$ which, as expected, do not change with $\theta_{c . m}$. However, such an angular dependent enhancement is not seen in the $\pi^{+}$and $\pi^{0}$ channels from the proton. The SAID FA09 prediction is 
also shown in Fig. 1 (blue curve), and it does show an enhancement around $\sqrt{s}$ of 2.2 $\mathrm{GeV}$ which is not angular dependent. Our studies show that such a behavior is not due to the FSI correction.

The observed angular dependent enhancement structure in the $\pi^{-}$channel could be due to some unknown resonances which couple differently to the neutron channel than to the proton channel. Polarization data from all three channels and partial wave analysis are necessary in order to understand the nature of this enhancement and its angular dependence in the $\pi^{-}$channel.

The data presented in this work are the first high statistical precision measurement of the differential cross section of the $\gamma n \rightarrow \pi^{-} p$ process in the region $\sqrt{s} \approx 1.8-2.5 \mathrm{GeV}$ with fine photon energy bins, and a pion center-of-mass angle between $50^{\circ}$ and $115^{\circ}$. Our data suggest a possible signature for the transition to the CCR scaling region, in the form of a fall-off of the scaled cross section over a narrow energy range. An angular dependent enhancement in the scaled differential cross section has been seen for the first time in our data, which is different from that of the $\gamma p \rightarrow \pi^{+} n$ process, and it is also different from the lastest SAID prediction.

\section{ACKNOWLEDGMENTS}

This work is supported in part by U.S. Department of Energy under contract DE-FG0203ER41231.

\section{REFERENCES}

1. L.Y. Zhu et al., Phys. Rev. Lett. 91, 022003 (2003).

2. L.Y. Zhu et al., Phys. Rev. C 71, 044603 (2005).

3. S.J. Brodsky and G.R. Farrar, Phys. Rev. Lett. 31, 1153 (1973); Phys. Rev. D 11, 1309 (1975); V. Matveev et al., Nuovo Cimento Lett. 7, 719 (1973).

4. G.P. Lepage and S.J. Brodsky, Phys. Rev. D 22, 2157 (1980).

5. R.L. Anderson et al., Phys. Rev. D 14, 679 (1976).

6. C. White et al., Phys. Rev. D 49, 58 (1994).

7. J. Napolitano et al., Phys. Rev. Lett. 61, 2530 (1988); S.J. Freedman et al., Phys. Rev. C 48, 1864 (1993); J.E. Belz et al., Phys. Rev. Lett. 74, 646 (1995).

8. C. Bochna et al., Phys. Rev. Lett. 81, 4576 (1998).

9. E.C. Schulte et al., Phys. Rev. Lett. 87, 102302 (2001).

10. M. Mirazita et al., Phys. Rev. C 70, 014005 (2004).

11. P. Rossi et al., Phys. Rev. Lett. 94, 012301 (2005).

12. W. Chen et al., Phys. Rev. Lett. 103, 012301 (2009)

13. B.A. Mecking et al., Nucl. Instr. and Meth. A 503, 513 (2003).

14. H. Gao, R.J. Holt, and V.R. Pandharipande, Phys. Rev. C 54, 2779 (1996).

15. J.M. Laget, Phys. Rev. C 73, 044003 (2006).

16. M. Dugger et al., e-print arXiv:0903.1110.

17. S.S. Kamalov et al., Phys. Rev. C 64, 032201(R) (2001) (A dynamical model); D. Drechsel et al., Nucl. Phys. A 645, 145 (1999) (a unitary isobar model); D. Drechsel, S.S. Kamalov, and L. Tiator, Euro. Phys. J. A34, 69 (2007).

18. A. Sibirtsev, J. Haidenbauer, S. Krewald, T.-S.H. Lee, U.-G. Meissner, A.W. Thomas, Euro. Phys. J. A34, 49 (2007); A. Sibirtsev, private communications.

19. M. Dugger et al., Phys. Rev. C 76, 025211 (2007). 